\title{
Multi-Objective Optimization of Activation Time and Discharge Time of Thermal Battery Using a Genetic Algorithm Approach
}

\author{
Qing $\mathrm{Li}^{1,2}$, Yu-Qiang Shao ${ }^{1}{ }^{1}$, Huan-Ling Liu ${ }^{1, * \mathbb{D}}$ and Xiao-Dong Shao ${ }^{1, *}$ \\ 1 School of Electromechanical Engineering, Xidian University, Xi'an 710071, China; xzxk8890@163.com (Q.L.); \\ qiangsy123@163.com (Y.-Q.S.) \\ 2 The 18th Research Institute of China Electronics Technology Group Corporation, Tianjin 300384, China \\ * Correspondence: hlliu@xidian.edu.cn (H.-L.L.); xdshao@xidian.edu.cn (X.-D.S.)
}

Received: 7 November 2020; Accepted: 4 December 2020; Published: 8 December 2020

\begin{abstract}
Activation time and discharge time are important criteria for the performance of thermal batteries. In this work a heat transfer analysis is carried out on the working process of thermal batteries. The effects of the thicknesses of heat pellets which are divided into three groups and that of the thickness of insulation layers on activation time and discharge time of thermal batteries are numerically studied using Fluent 15.0 when the sum of the thickness of heating plates and insulation layers remain unchanged. According to the numerical results, the optimal geometric parameters are obtained by using multi-objective genetic algorithm. The results show that the activation time is mainly determined by the thickness of the bottom heat pellet, while the discharge time is determined by the thickness of the heat pellets and that of the insulation layers. The discharge time of the optimized thermal battery is increased by $4.08 \%$, and the activation time is increased by $1.23 \%$.
\end{abstract}

Keywords: thermal batteries; activation time; discharge time; multi-objective genetic algorithm

\section{Introduction}

Thermal batteries, which are widely used in missiles and other modern weapons, have the advantages of long storage time, no self-discharge, quick activation and high reliability [1-4]. Unlike conventional batteries, thermal batteries need to operate at very high temperatures (usually more than $350^{\circ} \mathrm{C}[1,5]$ ) in order to melt the electrolyte, which is a solid at room temperature, which causes them to activate and discharge outward. How to shorten the activation time and prolong the discharge time is a popular research topic that has attracted wide attention. Some researchers have studied the activation stage of thermal batteries, such as Kang et al. [6], who numerically simulated the activation of thermal batteries without a centre hole, and concluded that heat conduction is the main factor affecting the activation time. Witt et al. [7] used a 3-dimensional scanning laser Doppler vibrometer (3-D SLDV) to capture the temperature variation of thermal batteries during activation process. Their results indicated that the electrical properties of thermal batteries vary with the temperature. Koyuncu et al. [8] established a two-dimensional finite element model based on COMSOL Multiphysics to study the activation time of thermal batteries. The model provided a reliable heat design tool for the activation phase of thermal batteries. For the discharge stage, Krieger et al. $[9,10]$ investigated the effects of gases and gas mixtures on global thermal conductivity values of porous thermal insulation packages of fully assembled low cost competent munition (LCCM) thermal batteries using transient and quasi-steady-state heat transfer techniques. Their results indicated that filling the thermal battery with an inert gas, such as krypton or xenon, significantly prolonged the discharge time of the thermal battery. Yang et al. [11] presented a one-dimensional mathematical model for a high 
temperature lithium-aluminum, iron disulfide molten salt battery system. The model predicts that increasing the battery's temperature can reduce the precipitation of $\mathrm{KCl}$ and prolong the discharge time. Haimovich et al. [12] proposed the method of using an additional buffer salt to prolong the discharge time. Chen et al. [13] developed a thermal battery which greatly prolongs the discharge time compared to the traditional thermal battery with a low melting point electrolyte. Jeong et al. [14] conducted thermal analysis on large-capacity thermal batteries, reporting that adding additional heat pellets to the insulation layer lead to longer discharge time.

Genetic algorithms (GAS) are a class of stochastic algorithms well-suited for large-scale optimization, which have been successfully applied to optimize lithium batteries heating strategy [15], multi-physical field modeling [16], and battery shell structure design [17]. The rationale of the GA procedure is used to simulate the process of natural selection and mutation and determine the optimal solution to minimize the errors between simulated data and experimental data.

Although many studie have been conducted in the simulation of the discharge phase and activation phase of thermal batteries, most of these studies have not studied these two phases as a whole. In addition, there is no research on optimizing the activation time and discharge time of thermal battery. However, rapid response devices require thermal batteries to have a short activation time and have a long work time the thermal battery work. In general, short activation time and long discharge time are two essential characteristics of high-performance thermal batteries. In the manufacture of thermal battery, the thickness of heat pellets should be increased to shorten the activation time, while the thickness of insulators should be increased to extend the discharge time. Obviously, both cannot be satisfied at the same time for a given shell. Therefore, in the actual design of thermal battery, the activation stage and discharge stage should be considered comprehensively, and the optimized structure parameters should be gotten in order to achieve the best performance of thermal batteries. It is urgent to investigate the relationship between the thickness of heat pellets and insulators in order to obtain the best activation time and discharge time. This work uses ANSYS Fluent to analyze the activation process and discharge process of the thermal battery and compares the data with that given in the literature to verify the correctness of the simulation results. From the perspective of shortening the activation time and increasing the discharge time, a multi-objective optimization method based on genetic algorithm is proposed. The thickness of the heat pellets in the electroactive battery and that of the insulation layers on the upper and lower sides are used as the decision variables to optimize the activation time and the discharge time. Finally, the optimization results are verified by simulation.

\section{Structural Composition and Parameters of Thermal Battery}

In this paper we use the thermal battery simulation model reported by Jeong et al. [14], as shown in Figure 1a. The 13-stack thermal battery is composed of a case, thermal insulation, top and bottom stack regions, and the electroactive cells, which consist of 13 layers of unit cells and 14 layers of heat pellets $\left(\mathrm{Fe} / \mathrm{KClO}_{4}\right)$. As shown in Figure 1c, the unit cell consists of cathode $\left(\mathrm{FeS}_{2}\right)$, electrolyte (LiF-LiCl-LiBr), anode (LiSi), and current collector (stainless steel). Stack regions with heat pellets and insulators A (mica), as shown in Figure 1b,d, are added to both ends of the electroactive cell to provide sufficient heat to both ends of the electroactive cell. 


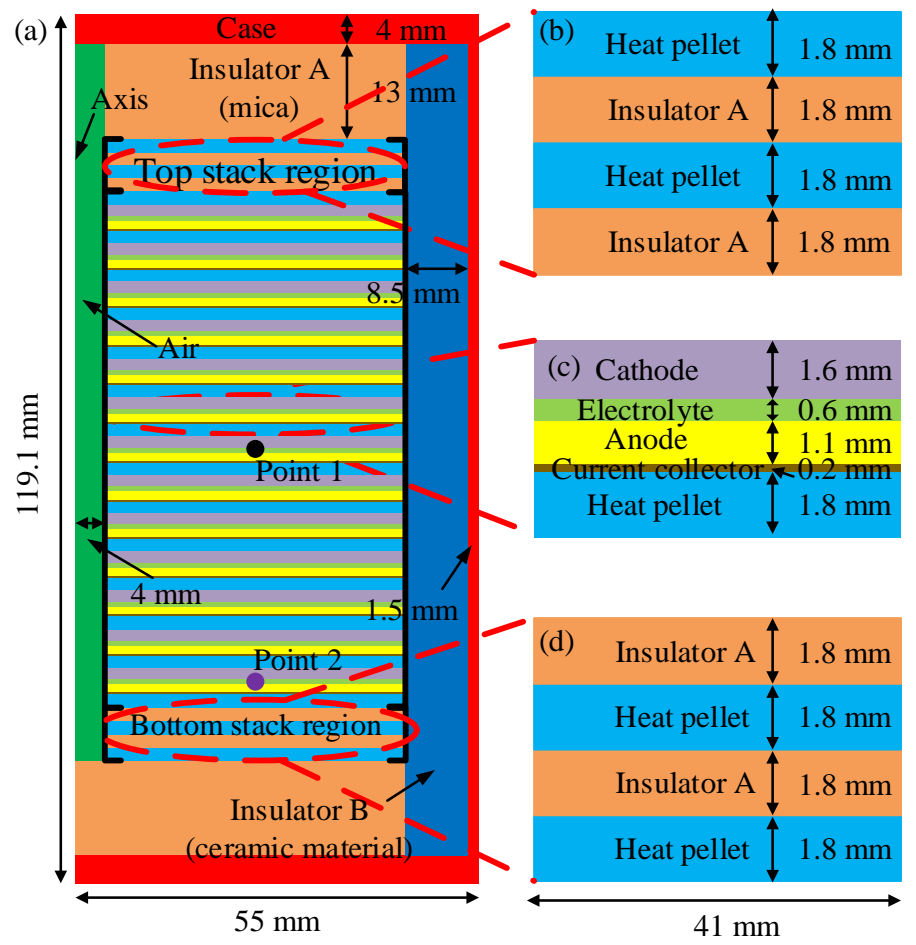

Figure 1. Schematic of (a) 13-stack thermal battery module, (b) top stack region, (c) unit cell and heat pellet, and (d) bottom stack region.

\section{Thermal Battery Simulation Model}

\subsection{Governing Equations and Boundary Conditions}

Assuming that the fluid is incompressible and the physical parameters are constant, the governing equations of the fluid are expressed as follows [18]:

Continuity equation:

$$
\nabla \cdot(\rho \vec{v})=0
$$

Momentum equation:

$$
\frac{\partial(\rho \vec{v})}{\partial t}+\nabla \cdot(\rho \vec{v} \vec{v})=-\nabla p+\mu \nabla^{2} \vec{v}
$$

Energy equation:

$$
\frac{\partial\left(\rho C_{p} T\right)}{\partial t}+\nabla \cdot\left(\rho C_{p} \vec{v} T\right)=\nabla \cdot(\lambda \nabla T)
$$

The boundary conditions are set as follows:

Initial temperature of the battery:

$$
T_{0}=300 \mathrm{~K} .
$$

At the wall of the battery:

$$
-\lambda\left(\frac{\partial T}{\partial n}\right)_{\text {wall }}=h\left(T_{\text {wall }}-T_{\mathrm{am}}\right)+\varepsilon \sigma\left(\left(\frac{T_{\text {wall }}}{100}\right)^{4}-\left(\frac{T_{\mathrm{am}}}{100}\right)^{4}\right),
$$

where $h=10 \mathrm{~W} \mathrm{~m}^{-2} \mathrm{~K}^{-1}$ [19], $T_{\mathrm{am}}=300 \mathrm{~K}, \varepsilon=0.11$ [19], $\sigma=5.67 \mathrm{~W} \mathrm{~m}^{-2} \mathrm{~K}^{-4}, n$ is the direction of outward normal.

At the axis:

$$
-\lambda\left(\frac{\partial T}{\partial n}\right)=0
$$


The thermophysical parameters for all materials are listed in Table 1.

Table 1. Thermophysical parameters at $300 \mathrm{~K}[6,20]$.

\begin{tabular}{|c|c|c|c|}
\hline Material & $\begin{array}{l}\text { Density/ } \\
\left(\mathrm{kg} \mathrm{m}^{-3}\right)\end{array}$ & $\begin{array}{c}\text { Specific/ } \\
\left(\mathrm{J} \mathrm{kg}^{-1} \mathrm{~K}^{-1}\right)\end{array}$ & $\begin{array}{l}\text { Thermal Conductivity/ } \\
\left(\mathrm{W} \mathrm{m}^{-1} \mathrm{~K}^{-1}\right)\end{array}$ \\
\hline Anode & 1050.04 & 2072.5 & 5.44 \\
\hline Electrolyte & 2210.47 & 870 & 1.02 \\
\hline Cathode & 2783 & 992.3 & 1.02 \\
\hline Current collector & 7937.08 & 690.8 & 21.65 \\
\hline Heat pellet & 3299.28 & 745.3 & 21.86 \\
\hline Insulator A (mica) & 2263.54 & 850 & 0.4 \\
\hline Insulator B (ceramic material) & 205.7 & 979.7 & 0.08 \\
\hline Case (stainless steel) & 8030 & 690.8 & 21.646 \\
\hline Air & 1.225 & 1006.4 & 0.024 \\
\hline
\end{tabular}

The commercial software FLUENT 15.0 is used as the simulation tool in this study. The heat paper is defined as the air, and the other computational domains are defined as the solid domain. The time step size can be divided by three. The time step of $0 \mathrm{~s}$ to $4 \mathrm{~s}$ is $0.005 \mathrm{~s}$, the time step of $4 \mathrm{~s}$ to $10 \mathrm{~s}$ is set as $0.1 \mathrm{~s}$ working as bumper to avoid the rapid change of time step size and after $10 \mathrm{~s}$, the time step is set as $5 \mathrm{~s}$, and the calculated time lasts until the end of the battery discharge time.

\subsection{Setting of Heat Source}

As previously mentioned, heat pellets burn to release energy, which melts the electrolytes when the thermal battery begins to discharge outwards. Assuming that heat pellets burn at the same time and produce heat evenly, the burning time $\Delta t_{h p}=0.42 \mathrm{~s}$ and the power density of a pellet $q_{h p}=9.7957 \times 10^{9} \mathrm{w} \mathrm{m}^{-3}$. When the electrolyte reaches its melting point $(709.15 \mathrm{~K})$ indicating that the thermal battery is activated. After the activation process, the Joule heating (exothermic) and chemical reaction heat (endothermic) are generated in the discharge phase. Joule heat is mainly generated at the cathode [14] and can be calculated by the following expression [21]:

$$
q_{j h}=i_{A}^{2} R,
$$

where $R$ is the resistivity and $i_{A}$ is the current per cross sectional area (i.e., current density). The resistivity of each component is $1.8 \times 10^{-4} \Omega \mathrm{m}$ for cathode [21], and the current density is $500 \mathrm{~A} \mathrm{~m}^{-2}$ [20]. On the other hand, chemical reaction heat is mainly generated at the cathode and anode [14] and can be estimated as [21]:

$$
q_{c r}=i_{V}\left(-T \frac{\partial U_{0}}{\partial T}\right)
$$

where $i_{V}$ is the current per unit volume (i.e., $i_{V}=i_{A} / \delta$ with $\delta$ being the radius of cell), $T$ is the temperature, and $\partial U_{0} / \partial T$ is the variation of the open-circuit cell potential with respect to temperature, $\partial U_{0} / \partial T=2 \times 10^{-4} \mathrm{~V} \mathrm{~K}^{-1}[21]$.

\subsection{Grid Independence Tests}

The thermal battery model is built by using Workbench 15.0, and the mesh is defined as a triangular grid. We test the mesh independence with four mesh systems of 229,$379 ; 272,758 ; 317,859$ and 811,353 . Figure 2 shows the average temperature change curve of the first layer electrolyte with different mesh systems. When the mesh number increases, the average temperature $\bar{T}_{\mathrm{e} 1}$ of the first layer electrolyte gradually increases. When the mesh number exceeds 317,859 , the value of $\bar{T}_{\mathrm{e} 1}$ tends to be consistent, and the maximum error is less than $0.1 \%$. Therefore, 317,859 is selected as the number of grid division in the simulation calculation when the accuracy meets the requirements. 


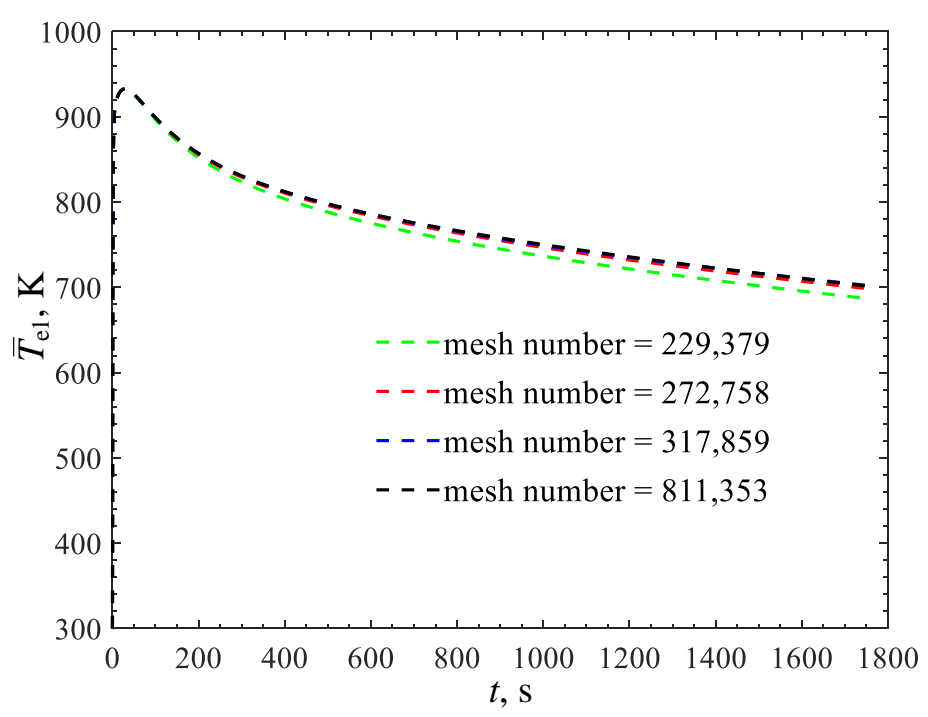

Figure 2. The curve of mesh independence verification.

\section{Numerical Simulation Results}

\subsection{Correctness Verification of Numerical Simulation}

In order to verify the correctness of the numerical simulation, we compare the calculated results with those of Jeong et al. [14]. Figure 3 shows the temperature curve of points 1 and 2 (as shown in Figure 1). It can be seen from Figure 3 that in the activation stage (0-1 s), the temperature in this paper is consistent with that in [14], so the activation time is not affected. In the discharge stage (after $1 \mathrm{~s}$ ), the temperature in this paper was slightly higher than that reported in [14], which would slightly increase the discharge time obtained in this paper. Although there are differences between the simulation results and [14], the variation trend and error of the results (the maximum relative error is $9.64 \%$ ) are within an acceptable range, and the optimization is carried out on the basis of the model established in this paper, that is to say, the optimization results are compared with the simulation results in this paper instead of [14]. Therefore, we conclude that the simulation error in this paper has no influence on the optimization result.

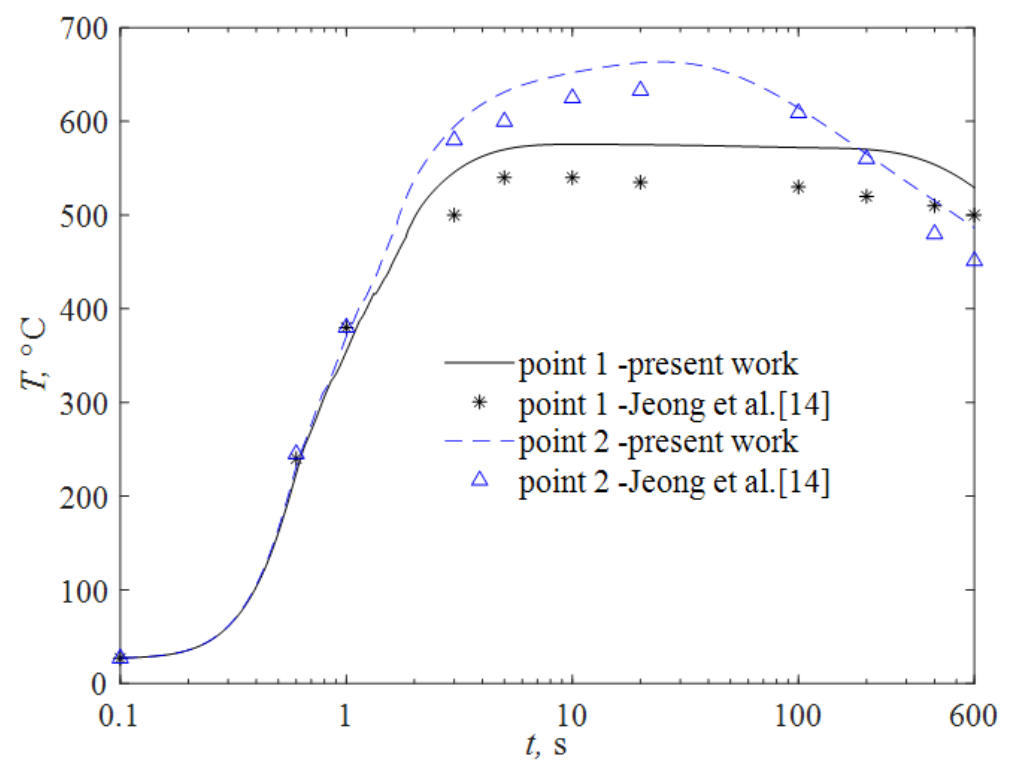

Figure 3. The comparison of the simulation results of this work with that of Jeong et al. [14]. 
4.2. Effects of Heat Pellet Thickness and Insulation Layer Thickness on the Activation Time and Discharge Time

As shown in Figure 4, the heat pellets are divided into three groups. The 1st to 4th layers are defined as group $a$, the 5th to 10th layers are deemed as group $b$, and the 11th to 14th layers are considered as group $c$. It is stipulated that the thickness of heat pellets in the same group is the same, and the total thickness of heat pellets and upper and lower insulation layers are constant, that is:

$$
d_{a t}=d_{a}+d_{b}+d_{c}+d_{i n s 1}+d_{i n s 2}
$$

where $d_{a t}$ is the total thickness of the heat pellets and the insulation layers on the upper and lower sides, $d_{a t}=51.2 \mathrm{~mm}$. $d_{a}, d_{b}$ and dc are the thicknesses of groups $a, b$ and $c$, respectively. Quantities $d_{i n s} 1$ and $d_{i n s 2}$ are the thickness of upper and lower insulation layers, respectively.

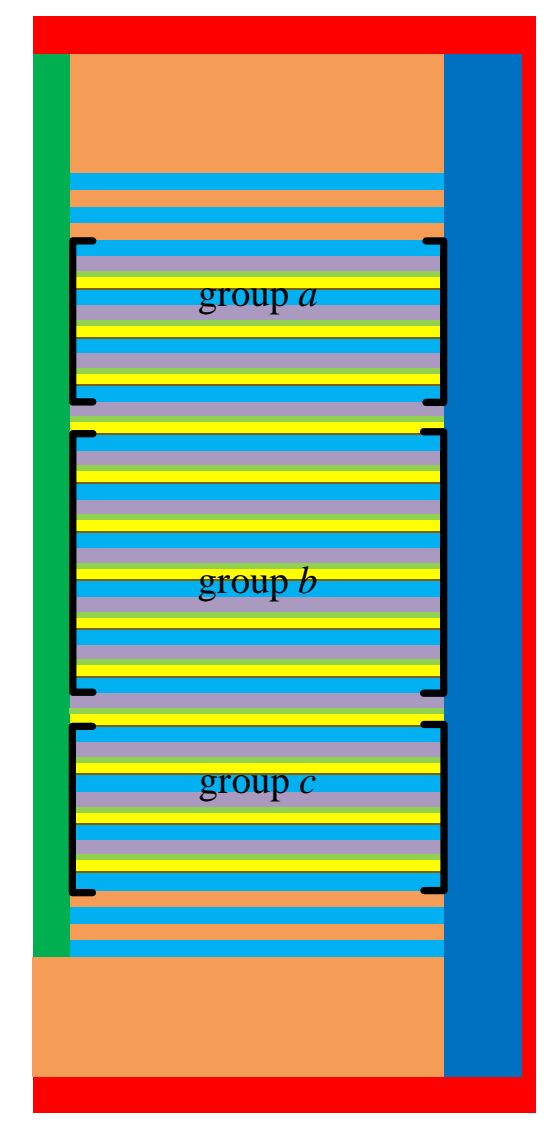

Figure 4. Sketch of the heat pellet group.

From Jeong et al. [14], it is known that the temperature of the 13th layer electrolyte decreases the fastest. In other words, the discharge time of the thermal battery is determined by the temperature of the 13th electrolyte layer. In order to extend the discharge time of the thermal battery, we can increase the thickness of the bottom insulation layer or the thickness of the bottom heat pellet. However, the battery temperature may be too high when the thickness of the bottom heat pellet is increased, resulting in the battery failing. Therefore, we increase $d_{i n s 2}$ by reducing $d_{a}$ and $d_{b}$ to achieve longer discharge time of the battery, namely:

$$
\Delta d_{\text {ins } 2}=-\left(\Delta d_{a}+\Delta d_{b}\right),
$$

where $\Delta d_{a}$ and $\Delta d_{b}$ are the decrease of the thickness of group $a$ and $b$ respectively, and $\Delta d_{\text {ins } 2}$ is the thickness difference of the insulation layer at the bottom. 
For a thermal battery to reach full discharge phase, the temperature of the electrolyte should always be above its melting point. In order to study the effect of the thickness of the heat pellets on the temperature of the electrolyte, simulations are performed for the thermal battery with different $\Delta d_{a}$ and $\Delta d_{b}$.

Figure 5 is the temperature profile of the inner part of the thermal battery module at $t=1250 \mathrm{~s}$ for different thicknesses, which shows the temperature of the mid-point of the thirteenth layer (the layer has the lowest temperature). It can be seen that the internal temperature at the middle of the battery is high while that at both sides is low. From Figure $5 \mathrm{a}-\mathrm{c}$, with increasing $\Delta d_{b}$, the temperature of the 13th layer electrolyte increases, which indicates that reducing the thickness of the heat pellets of group $b$ may extend the discharge time of the thermal battery. When $\Delta d_{b}=0.24 \mathrm{~mm}$, the temperature of electrolyte increases obviously. This is because the heat pellets become thinner leading to less heat released. However, the heat loss is reduced when the thickness of the insulation is increased, while resulting in a higher battery temperature. It is determined by the heat production of heat pellets and the insulation effect of insulation layers. When $\Delta d_{a}=0.36 \mathrm{~mm}$, the mechanism is just the opposite when the heat production of heat pellets plays a decisive role.

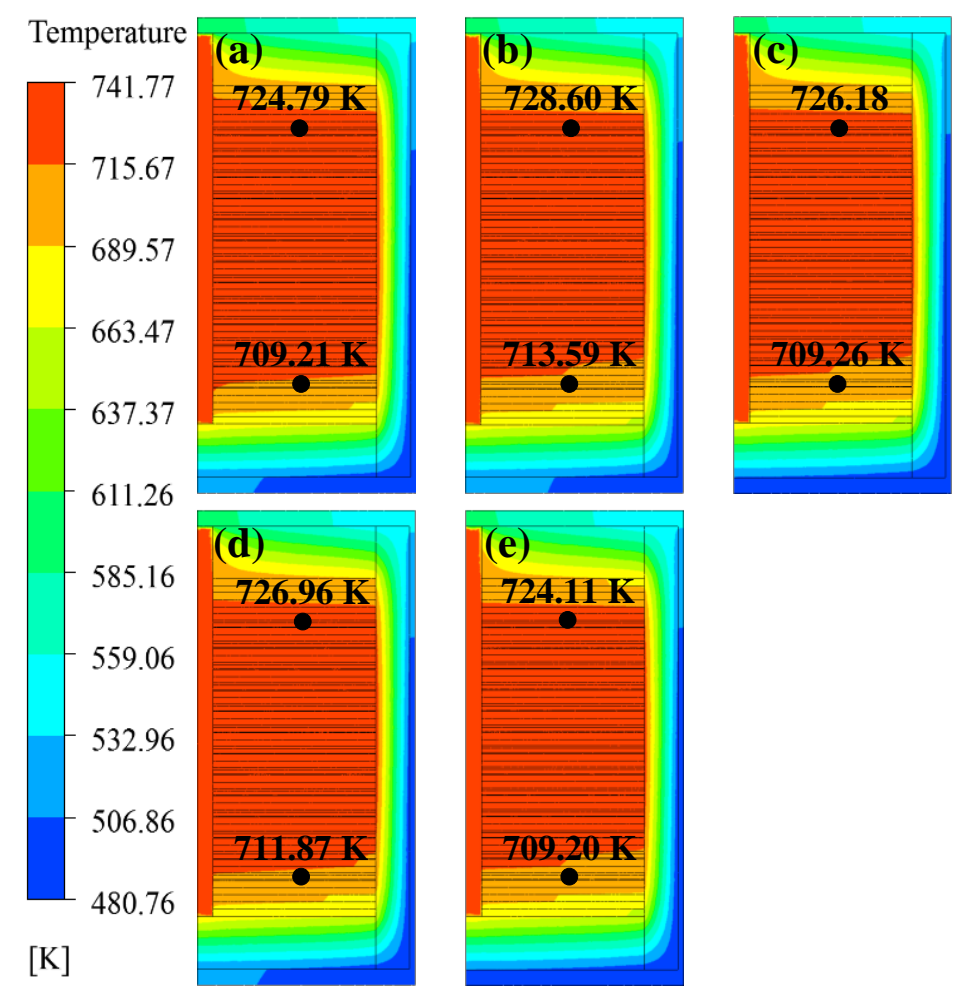

Figure 5. Temperature contours of the thermal battery module for (a) $\Delta d_{a}=0, \Delta d_{b}=0$; (b) $\Delta d_{a}=0$, $\Delta d_{b}=0.24 \mathrm{~mm}$; (c) $\Delta d_{a}=0, \Delta d_{b}=0.36 \mathrm{~mm}$; (d) $\Delta d_{a}=0.12 \mathrm{~mm}, \Delta d_{b}=0 ;(\mathbf{e}) \Delta d_{a}=0.24 \mathrm{~mm}, \Delta d_{b}=0$ at $t=1250 \mathrm{~s}$.

From Figure $5 \mathrm{a}$,d, it can be seen that the temperature of the 13th layer electrolyte can be increased by reducing the thickness of the heat pellets of group $a$. Comparing Figure $5 \mathrm{a}$,e, we find that the temperature of the 13th layer electrolyte is actually decreased when the thickness of the heat pellets in group $a$ is reduced by $0.24 \mathrm{~mm}$. This is because heat pellets become thinner, resulting in less heat generated. At this time, the insulation layer's heat preservation effect is insufficient to compensate for the heat loss caused by the thinning of the heat pellets.

Figure 6a shows the influence of the thickness of group $a$ for heat pellets on the time corresponding to the start of solidification of each layer electrolyte. The electrolyte on both sides of the electroactive cells is solidified before the middle part, and the 13th layer electrolyte is solidified firstly, which exhibits 
that the discharge time of the thermal battery is determined by the solidification time of the 13th layer electrolyte, that is $t_{1,13}=t_{\mathrm{e}-\mathrm{d}}$. When $\Delta d_{a}=0.12 \mathrm{~mm}$, the solidification time of the 13th layer electrolyte is $1290 \mathrm{~s}$, which indicating that the discharge time of the thermal battery is $1290 \mathrm{~s}$. Compared with the results given in Ref. [14] (the discharge time of the thermal battery is $1250 \mathrm{~s}$ ), the discharge time of this work is increased by $40 \mathrm{~s}$. When $\Delta d_{a}=0.24 \mathrm{~mm}$, the discharge time is $1257 \mathrm{~s}$. It also can be observed that the discharge time is nonlinear with $\Delta d_{a}$. This can be explained that the thickness of the heat pellets becomes thinner leading to a thicker insulation layer which slows the heat exchange between the battery and the air. Therefore, the time for the electrolyte to solidify is prolonged. Figure $6 \mathrm{~b}$ shows the influence of the thickness of group $b$ heat pellets on the time. It can be seen that when $\Delta d_{b}=0.24 \mathrm{~mm}$, the solidification time of the 13th layer electrolyte is $1293 \mathrm{~s}$, namely, the discharge time increases by $43 \mathrm{~s}$. When $\Delta d_{b}=0.36 \mathrm{~mm}$, the discharge time is $1265 \mathrm{~s}$, which shows that the discharge time is also nonlinear with $\Delta d_{b}$.

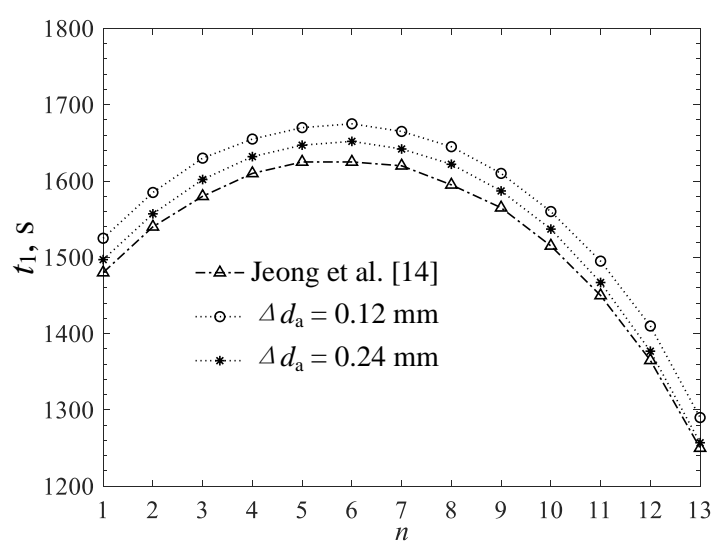

(a)

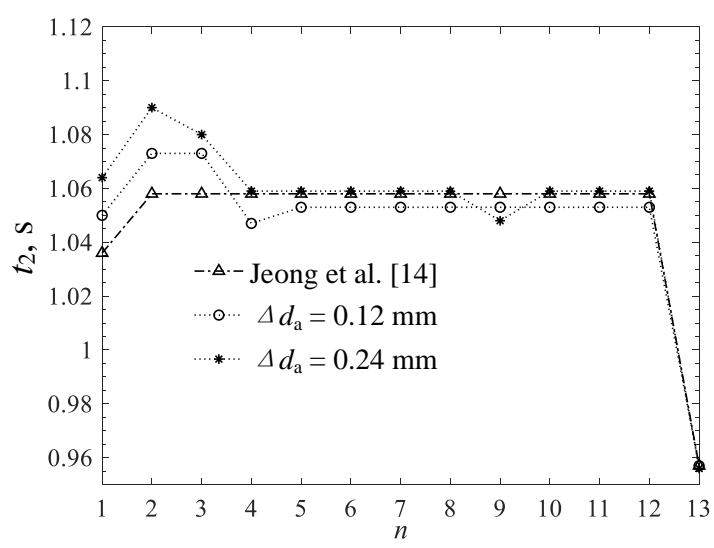

(c)

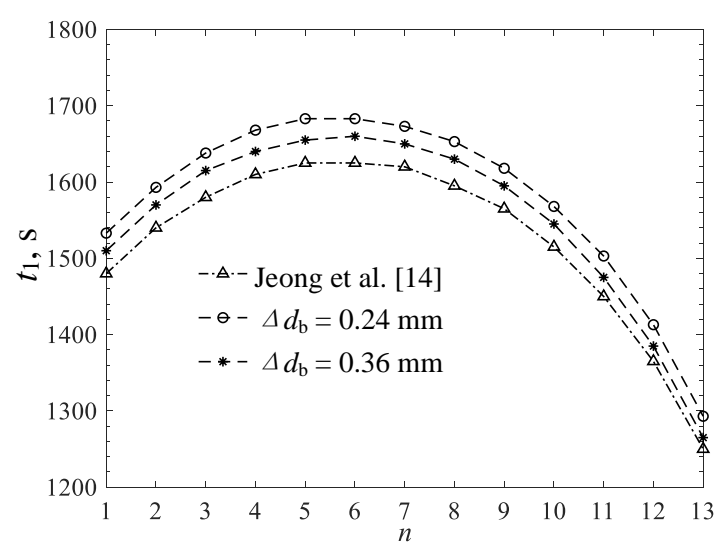

(b)

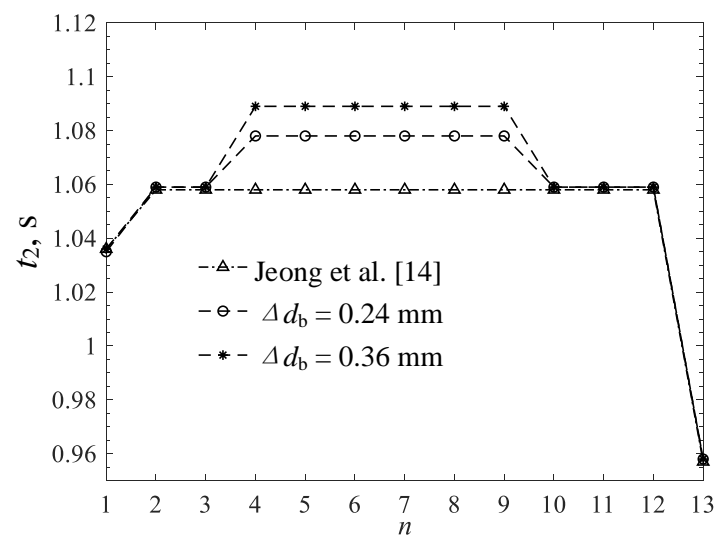

(d)

Figure 6. The influence of the thickness on the time corresponding to the initial solidification of each layer electrolyte for: (a) group $a$, and (b) group $b$ heat pellets on the; the influence of the thickness on the time of complete melting of each layer electrolyte for: (c) group $a$, and (d) group $b$ heat pellets.

Figure $6 c$ shows the effect of the thickness of group $a$ heat pellets on the melting time for all layers of electrolyte. It can be seen that the 2nd layer electrolyte finally melts when the time is $1.073 \mathrm{~s}$ for $\Delta d_{a}=0.12 \mathrm{~mm}$. This can be considered that the activation time of the thermal battery is $1.073 \mathrm{~s}$, namely, $t_{a c t i}=t_{2,2}=1.073 \mathrm{~s}$. The activation time is longer than that given in Ref. [14] (the activation time of the thermal battery is $1.058 \mathrm{~s}$ ). This indicates that reducing the thickness of the heater can increase the activation time It can also be seen that the larger $\Delta d_{a}$ is, the longer activation time of the thermal battery is. This is because the total heat is released when decreases the thickness of the heat pellet. As a result, 
the heat absorbed by the unit cell decreases, the rate of electrolyte melting decreases, and the activation time increases. Figure $6 \mathrm{~d}$ shows the influence of the thickness of group $b$ heat pellets on the time. It can be seen that when $\Delta d_{b}=0.24 \mathrm{~mm}$, the electrolytes of layers four to nine melt almost simultaneously, and the time for complete melting is $1.078 \mathrm{~s}$, that is to say, the activation time is increased by $0.02 \mathrm{~s}$. It can also be seen that the larger the $\Delta d_{b}$ is, the longer the activation time of the thermal battery is. This is because the heat pellets adjacent to these electrolytes become thinner, resulting in a decrease in heat absorbed by the electrolytes, which causes an increase in activation time.

In the actual design process of thermal battery, the activation time should be as short as possible while the discharge time should be as long as possible. Therefore, it is necessary to comprehensively consider the relationship between the activation time and the discharge time to get the optimized $\Delta d_{a}$ and $\Delta d_{b}$ to meet the needs of the project. The specific optimization details are given in Section 5.

\section{Optimization Details}

\subsection{The Theoretical Basis of Genetic Algorithm}

Based on MATLAB function fitting toolbox and genetic algorithm toolbox, we use $\Delta d_{a}$ and $\Delta d_{b}$ to undertake independent variables. Moreover, the discharge time $t_{1}$ and activation time $t_{2}$ are chosen as the objective functions in the process of the genetic algorithm. The flowcharts of algorithm of fitting function and GA technique are shown in Figure 7.

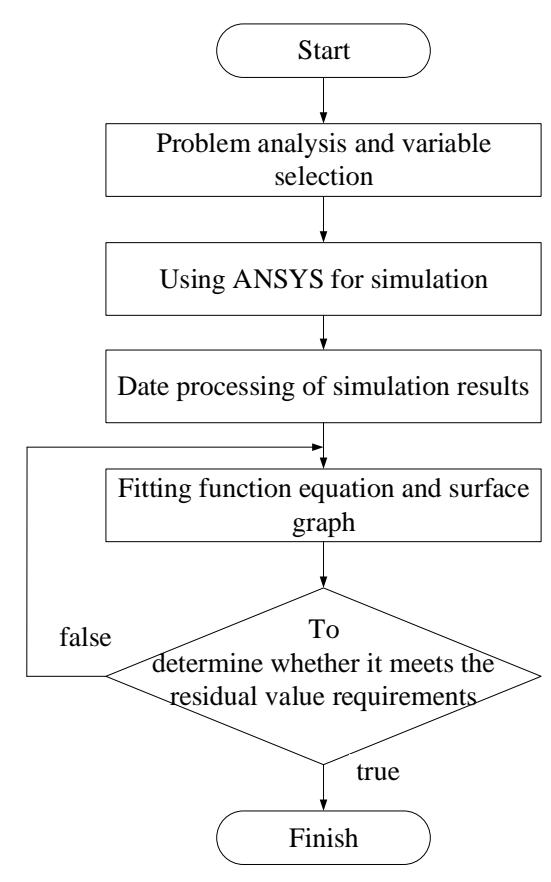

(a)

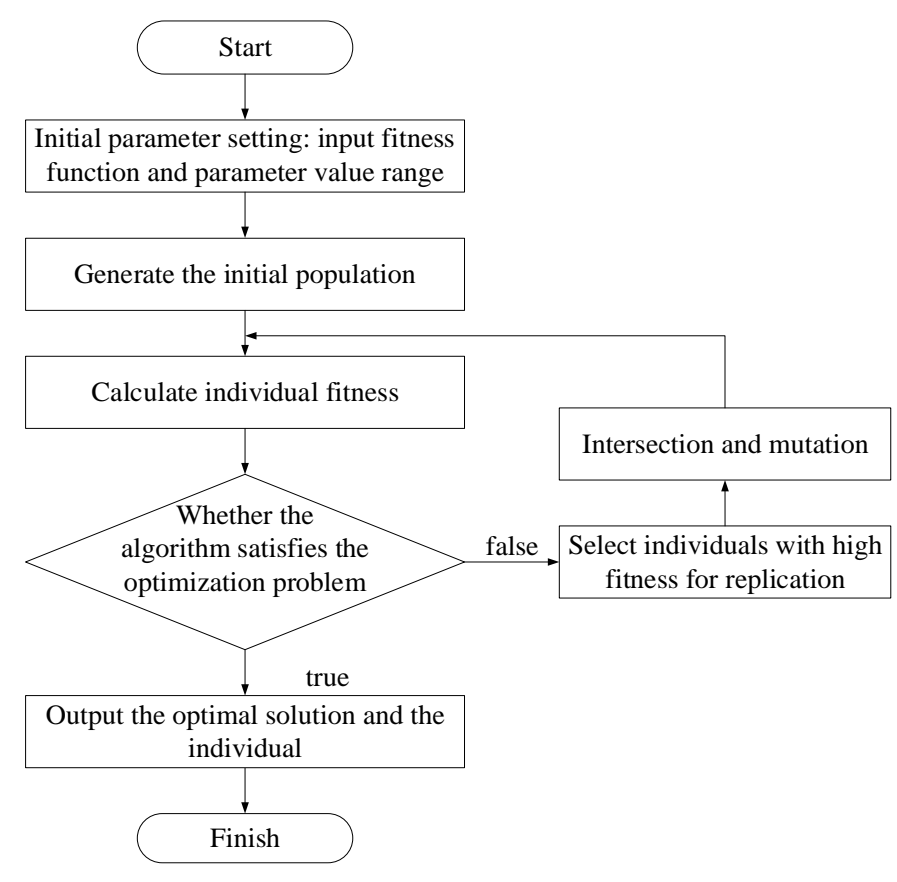

(b)

Figure 7. Optimization process of genetic algorithm: (a) flow chart of fitting function; (b) flow chart of genetic algorithm.

\subsection{The Generation of the Fitting Function}

Two design variables are used to derive the expression of $t_{a c t i}$ and $t_{e-d}$ for $\Delta d_{a}=0,0.12,0.16,0.2$, 0.24 and $0.32 \mathrm{~mm}$ when $\Delta d_{b}$ equals $0,0.18,0.24,0.3$ and $0.36 \mathrm{~mm}$, respectively. The most common method to solve the curve fitting problem is the least square method. By observing the simulation data (shown in Figure 8) and referring to the simulation work of ref. [18], we find that the change law of these data satisfies the polynomial fitting type. In addition, the R-square $\left(R^{2}=0.9681\right.$ and 0.9929$)$ and root mean square error $(R M E S=0.0074$ and 0.0011$)$ of the fitting function meet the requirements of 
high-quality fitting, which further proves that the selected fitting type is appropriate. Rewritten $\Delta d_{a} / H$ as $x_{1}, \Delta d_{b} / H$ as $x_{2}$ when $H=1 \mathrm{~mm}$, we have the following expressions:

$$
\begin{aligned}
& y_{1}=\frac{t_{e-d}}{t_{e-d, x_{1}=x_{2}=0}}=0.9969-1.003 \times x_{1}+0.3676 \times x_{2}+22.61 \times x_{1}{ }^{2}-8.528 \times x_{1} \times x_{2} \\
& -0.5282 \times x_{2}^{2}-124.9 \times x_{1}{ }^{3}+39.43 \times x_{1}{ }^{2} \times x_{2}+7.392 \times x_{1} \times x_{2}{ }^{2}-1.087 \times x_{2}{ }^{3} \\
& +202 \times x_{1}{ }^{4}-82.12 \times x_{1}{ }^{3} \times x_{2}+16.47 \times x_{1}{ }^{2} \times x_{2}{ }^{2}-5.44 \times x_{1} \times x_{2}{ }^{3} \\
& y_{2}=\frac{t_{a c t i}}{t_{\text {acti }, x_{1}=x_{2}=0}}=1+0.00921 \times x_{1}+0.08403 \times x_{2}+1.261 \times x_{1}{ }^{2}-0.7122 \times x_{1} \times x_{2} \\
& -0.0964 \times x_{2}{ }^{2}-4.697 \times x_{1}{ }^{3}-0.2079 \times x_{1}{ }^{2} \times x_{2}+1.685 \times x_{1} \times x_{2}{ }^{2}+0.2651 \times x_{2}{ }^{3} \\
& +5.871 \times x_{1}{ }^{4}+6.412 \times x_{1}{ }^{3} \times x_{2}-6.638 \times x_{1}{ }^{2} \times x_{2}{ }^{2}+0.2205 \times x_{1} \times x_{2}{ }^{3}
\end{aligned}
$$

where $t_{e-d}, x_{1}=x_{2}=0$ is the discharge time of the thermal battery when $x_{1}=x_{2}=0$, while $t_{\text {acti, } x_{1}=x_{2}=0}$ is the activation time when $x_{1}=x_{2}=0$. The value of $t_{e-d, x_{1}=x_{2}=0}$ is $1255 \mathrm{~s}$ and that of $t_{a c t i, x_{1}=x_{2}=0}$ is $1.06 \mathrm{~s}$.

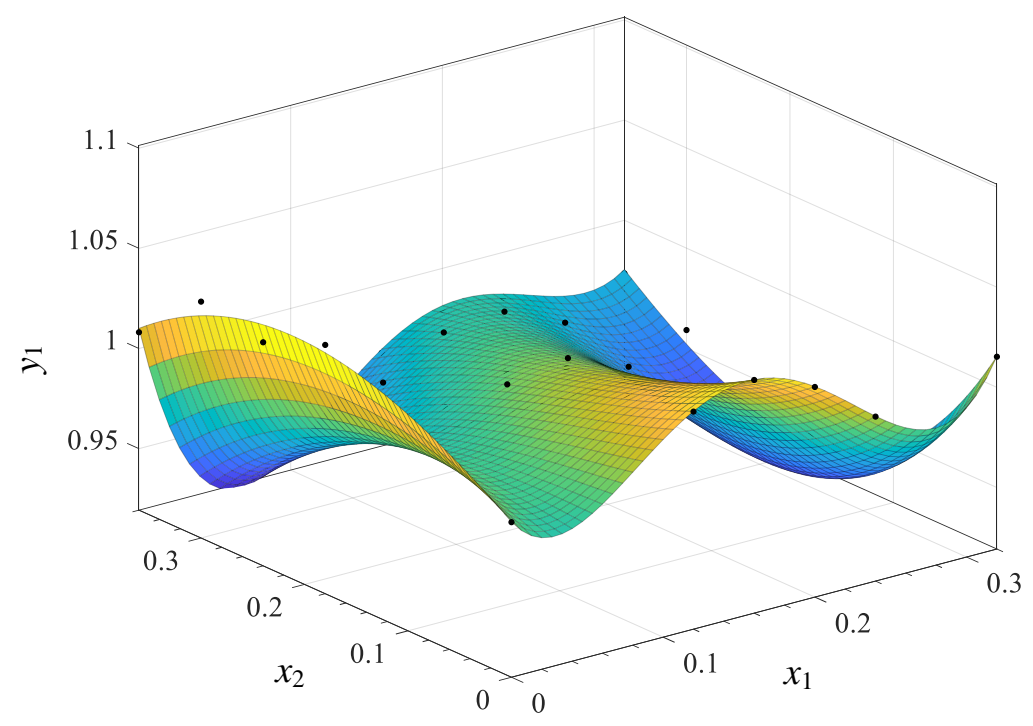

(a)

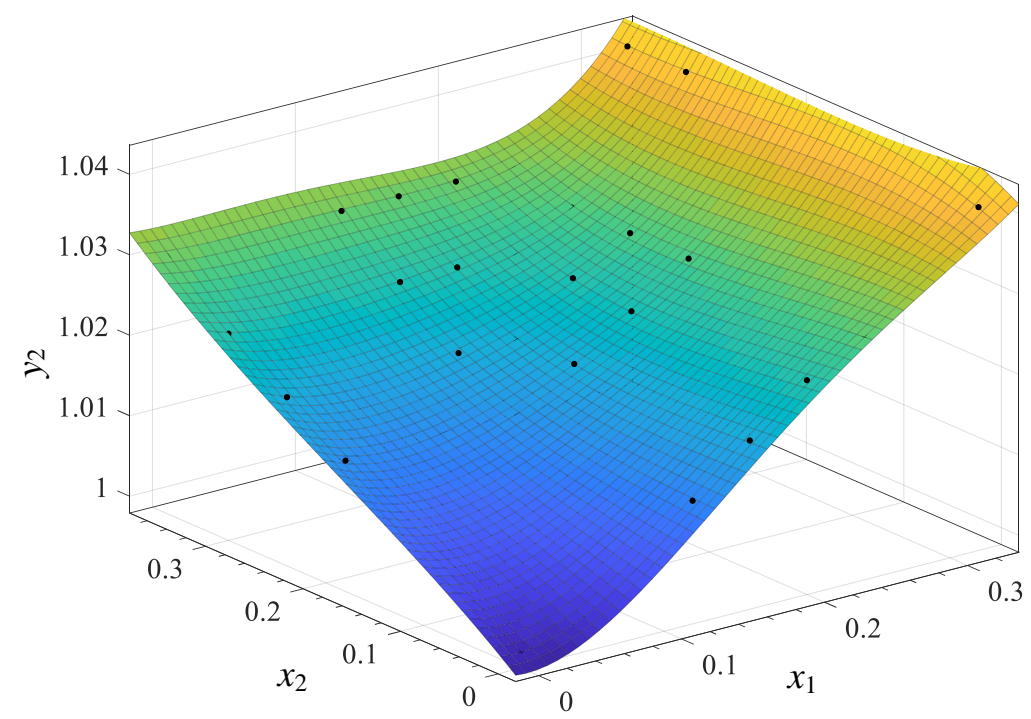

(b)

Figure 8. Fitting surface: (a) fitting surface of $y_{1}$; (b) fitting surface of $y_{2}$. 
R-square (adj. $\left.R^{2}\right)$, root mean square error $(R M S E)$ and Theil-U factor $\left(U_{2}\right)$ [22] are used to determine the accuracy of the above polynomials and to predict the discharge time and activation time of the thermal battery:

$$
\begin{gathered}
R^{2}=1-\frac{\sum_{i}\left(N_{i}-P_{i}\right)^{2}}{\sum_{i}\left(N_{i}-\overline{N_{i}}\right)^{2}}, \\
R M S E=\sqrt{\frac{1}{n} \sum_{i}\left(N_{i}-P_{i}\right)^{2}}, \\
U_{2}=\frac{\left[1 / n \sum_{i}\left(N_{i}-P_{i}\right)^{2}\right]^{0.5}}{\left[1 / n \sum_{i}\left(N_{i}\right)^{2}\right]^{0.5}},
\end{gathered}
$$

where $N_{i}$ is the control volume output, $\bar{N}_{i}$ is the average of control volume output, $P_{i}$ is the predicted value, and $n$ is the total number of data. The comparison of the predicted $y_{1}$ and $y_{2}$ with numerical results are shown in Figure 8a,b. The value of $R^{2}$ for $y_{1}$ is 0.9681 , and that of $R M S E$ and $U_{2}$ is 0.0074 and 0.00756 respectively. $R^{2}=0.9929, R M S E=0.0011$ and $U_{2}=0.00107$ for $y_{2}$, which reveals good agreements between the predicted values and the CFD data.

\subsection{Multi-Objective Optimization}

From the above study, it can be found that reducing the thickness of the heat pellets can prolong the discharge time of the thermal battery, which inevitably leads to an increase in the activation time. Therefore, multi-objective optimization of the activation time and discharge time is needed. According to Equations (11) and (12), we need find the maximum value of $y_{1}$ and the minimum value of $y_{2}$. Therefore, consider the following objective function:

$$
z=\left\{\begin{array}{c}
\operatorname{Min} 1 / y_{1} \\
\operatorname{Min} y_{2}
\end{array}, \text { subject to } 0 \leq x_{1} \leq 0.32,0 \leq x_{2} \leq 0.36\right.
$$

It is very important for industrial production to choose the best solution to meet the engineering requirements (i.e., shorten the activation time and extend the discharge time) from the optimization results.

As solutions shown in Pareto fronts are non-dominated, it is necessary to selected the best solutions from Pareto fronts according to their actual applications. TOPSIS [23-25] method is adopted to select the optimal solution. The basic principle is that if the solution is the closest to the positive ideal solution (i.e., solution with the minimum $1 / y_{1}$ and $y_{2}$ ) and the furthest away from the negative ideal solution (i.e., solution with the maximum $1 / y_{1}$ and $y_{2}$ ), it is the best. Otherwise, it is not optimal. The primary steps are as follows [26]:

(1) Create a normalized decision-making matrix $\left(t_{i j}\right)_{m \times n}$ by vector normalization:

$$
t_{i j}=\frac{x_{i j}}{\sqrt{\sum_{i-1}^{m} x_{i j}^{2}}} .
$$

where $m$ and $n$ are the number of solutions and objective functions, respectively. $x_{i j}$ is the element of matrix.

(2) Constitute a weighted normalized matrix:

$$
a_{i j}=w_{j} \times t_{i j}
$$


where $w_{j}$ is the weighting coefficient.

(3) Determine the positive and negative ideal solutions, $A^{+}$and $A^{-}$:

$$
\begin{aligned}
& A^{+}=\left(\min \left[a_{11}, \ldots, a_{m 1}\right], \min \left[a_{12}, \ldots, a_{m 2}\right], \ldots, \min \left[a_{1 n}, \ldots, a_{m n}\right]\right), \\
& A^{-}=\left(\max \left[a_{11}, \ldots, a_{m 1}\right], \max \left[a_{12}, \ldots, a_{m 2}\right], \ldots, \max \left[a_{1 n}, \ldots, a_{m n}\right]\right) .
\end{aligned}
$$

(4) Calculate the distance of each non-dominated solution to the positive and negative ideal solutions:

$$
\begin{aligned}
& d_{i}^{+}=\sqrt{\sum_{j=1}^{n}\left(a_{i j}-A_{j}^{+}\right)^{2}} . \\
& d_{i}^{-}=\sqrt{\sum_{j=1}^{n}\left(a_{i j}-A_{j}^{-}\right)^{2}} .
\end{aligned}
$$

(5) Calculate the closeness $c_{i}$ of each non-dominated solution to the positive ideal solution:

$$
c_{i}=\frac{d_{i}^{-}}{d_{i}^{+}+d_{i}^{-}}
$$

(6) Rank the $c_{i}$, and the best solution is

$$
A_{\text {best }}=A \in \max \left(c_{i}\right)
$$

In the MATLAB toolbox, we use the gamultiobj solver to optimize the expression. In the multi-objective optimization algorithm, the size of population $(P)$ and generation $(G)$ are key parameters affecting the obtained Pareto front. Sensitivity analysis of these two parameters is carried out. Figure 9 is the Pareto front obtained by gamultiobj with different population and generations. It can be seen that with the increase of generation, the Pareto front gradually converges to the same position. The greater the population, the more extensive the distribution of Pareto front. Considering the influences of the value of population and generations, the trial with parameters $P=50, G=400$ has the best performance among all trials, which is used as the obtained Pareto front as showed in Figure 10. The parameters are shown in Table 2. In order to verify the stability of the optimization program, the program is run several times. Figure 10 shows the multi-objective optimization results of four runs. The running results are almost consistent, which proves the stability of the optimized program. It can be seen that $y_{2}$ is negatively correlated with the reciprocal of $y_{1}$. That is to say, the longer activation time is, the longer discharge time is. However, we need reduce the activation time and increase the discharge time. The points in the graph are all non-inferior solutions, which has smaller target conflicts than other solutions. Table 3 shows all non-inferior solutions and structural parameters for the fourth run. When the activation time is not more than $1.075 \mathrm{~s}$, we select $x_{1}=0$ and $x_{2}=0.161$, that is, $\Delta d_{a}=0$, $\Delta d_{b}=0.161 \mathrm{~mm}$, the optimization result is the best.

Table 2. Parameters associated with gamultiobj.

\begin{tabular}{cc}
\hline Parameter & Algorithm or Value \\
\hline Population size & 50 \\
Generation & 400 \\
Pool size & 100 \\
Crossover and mutation & Scattered \\
Crossover fraction & 0.8 \\
Tournament size & 2 \\
\hline
\end{tabular}




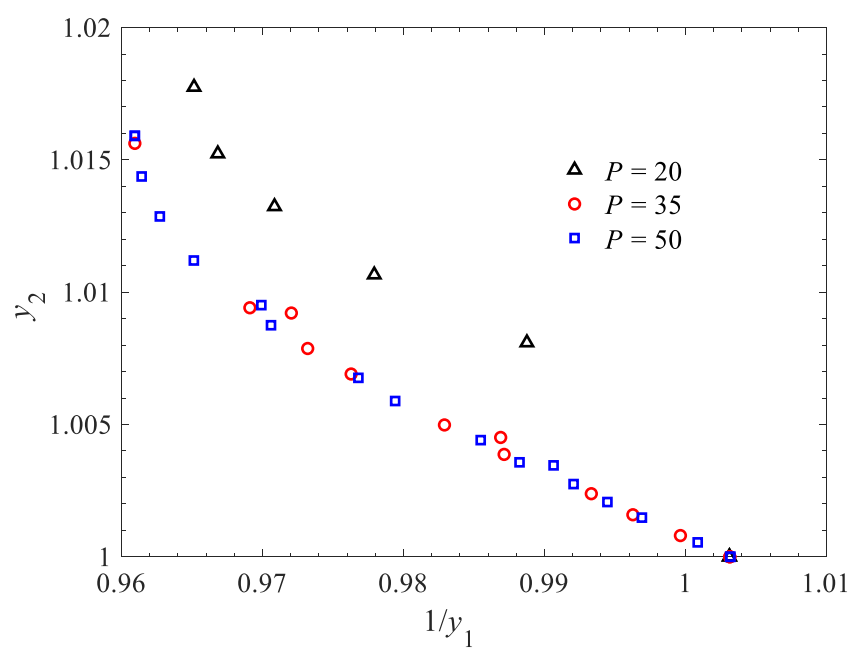

(a)

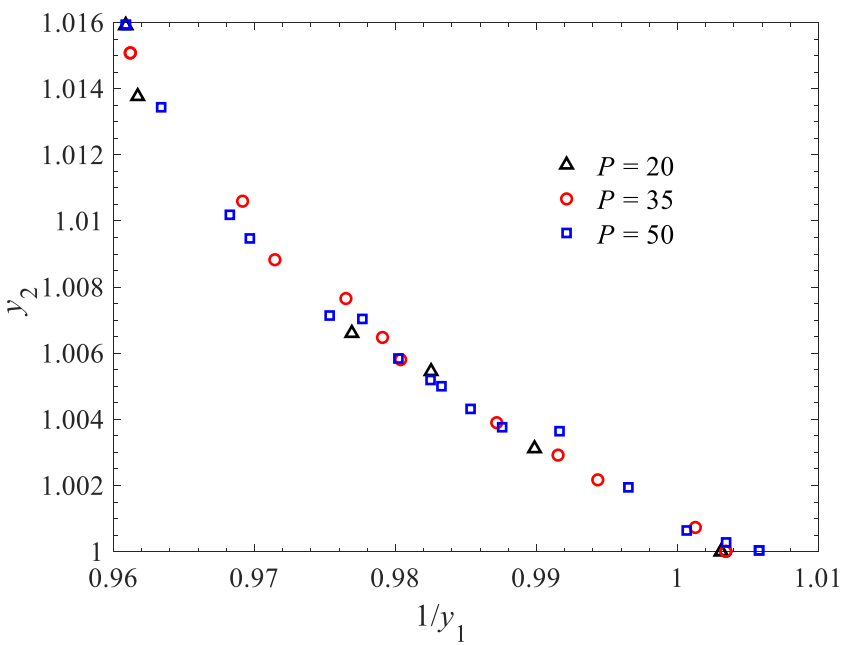

(b)

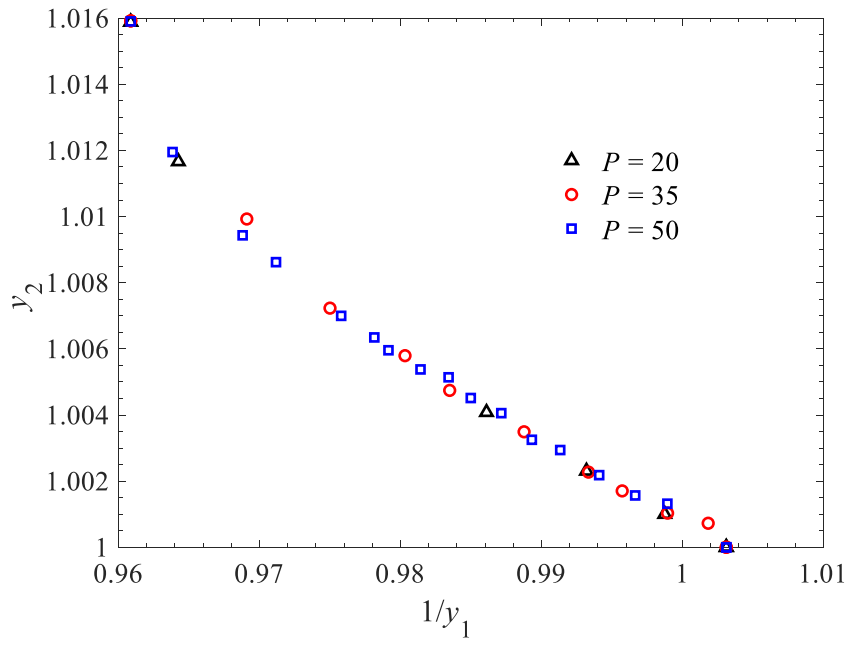

(c)

Figure 9. The Pareto front obtained by gamultiobj with (a) $G=200 ;(\mathbf{b}) G=300$; (c) $G=400$. 


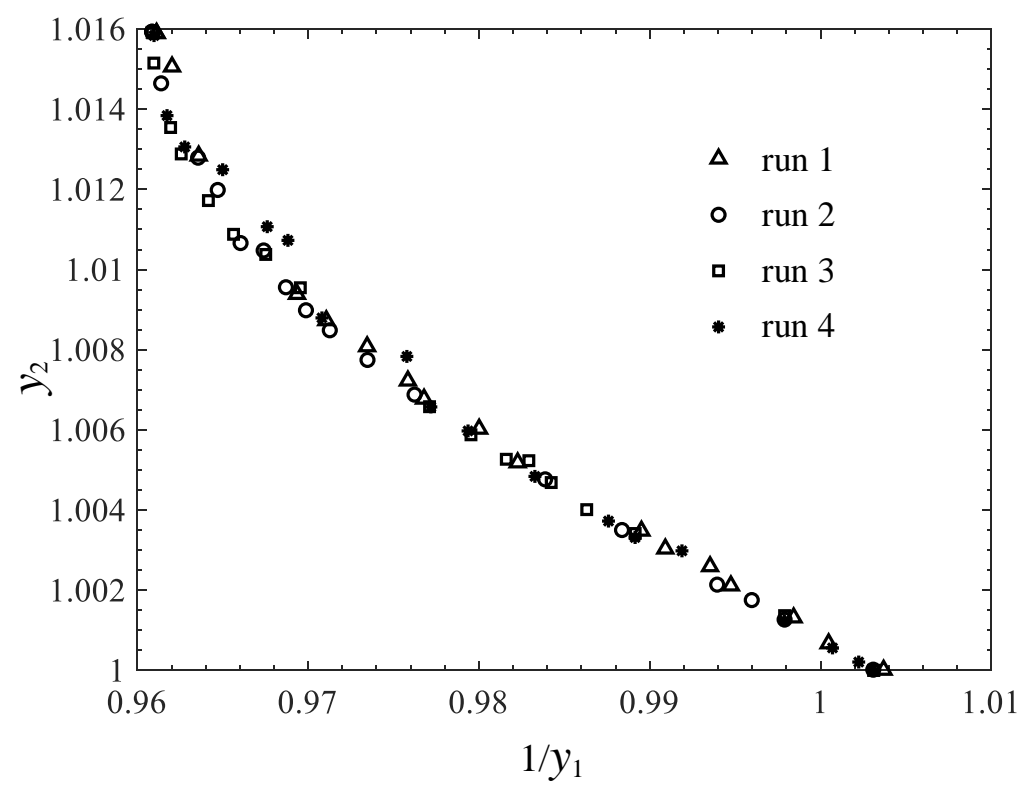

Figure 10. Distribution of Pareto fronts for four runs.

Table 3. The structural parameters corresponding to noninferior solutions.

\begin{tabular}{cccc}
\hline$x_{\mathbf{1}}$ & $\boldsymbol{x}_{\mathbf{2}}$ & $\boldsymbol{t}_{\boldsymbol{e}-\boldsymbol{d}}(\mathbf{s})$ & $\boldsymbol{t}_{\boldsymbol{a c t i}} \mathbf{( s )}$ \\
\hline 0.000 & 0.000 & 1246 & 1.06 \\
0.000 & 0.211 & 1301 & 1.077 \\
0.000 & 0.000 & 1246 & 1.06 \\
0.000 & 0.211 & 1301 & 1.077 \\
0.000 & 0.161 & 1297 & 1.073 \\
0.000 & 0.012 & 1251 & 1.061 \\
0.000 & 0.059 & 1270 & 1.065 \\
0.000 & 0.049 & 1266 & 1.064 \\
0.000 & 0.057 & 1269 & 1.065 \\
0.001 & 0.133 & 1290.866 & 1.0706459 \\
0.000 & 0.118 & 1288.663 & 1.069512975 \\
0.000 & 0.006 & 1248.644 & 1.060505051 \\
0.001 & 0.066 & 1272.049 & 1.06552876 \\
0.000 & 0.082 & 1278.719 & 1.066806676 \\
0.000 & 0.103 & 1284.469 & 1.068378362 \\
0.000 & 0.030 & 1259.167 & 1.062611205 \\
0.000 & 0.026 & 1256.746 & 1.062217672 \\
0.001 & 0.152 & 1294.601 & 1.072145892 \\
\hline
\end{tabular}

\subsection{Comparison of Optimization Results and Simulation Results}

When $\Delta d_{a}=0, \Delta d_{b}=0.161 \mathrm{~mm}$, the maximum error between the optimization results and the simulation results is $0.384 \%$. According to Table 4, after optimizing the thickness of heat pellets and insulation layer, the discharge time is increased by $51 \mathrm{~s}$, while the activation time only is increased by $0.013 \mathrm{~s}$, which greatly improves the use efficiency of thermal battery.

Table 4. Comparison of the results between simulation and multi-objective optimization when $\Delta d_{a}=0$, $\Delta d_{b}=0.161 \mathrm{~mm}$.

\begin{tabular}{cccc}
\hline Parameters & Multi-Objective Optimization & CFD Results & Relative Error \\
\hline$t_{a c t i}[\mathrm{~s}]$ & 1.073 & 1.075 & $0.189 \%$ \\
$t_{e-d}[\mathrm{~s}]$ & 1297 & 1302 & $0.384 \%$ \\
\hline
\end{tabular}




\section{Conclusions}

The active and discharge performance of a thermal battery is investigated numerically. Compared with [14], the correctness of the numerical results is verified. Under the condition that the total thickness of heat pellets and insulation layers remains unchanged, the influence of the thickness of each heat pellet and upper and lower insulation layer (i.e., if the heat pellets become thicker, the insulation layers will become thinner; if the heat pellets become thinner, the insulation layers will become thicker) on the discharge time and activation time of thermal battery is studied. The optimal geometric parameters of thermal battery are obtained by multi-objective optimization of thermal battery by genetic algorithm. From the results the following conclusions are made:

(1) The activation time and discharge time of thermal battery are related to the thickness of heat pellets and insulation layers. Adjusting the activation time and discharge time can be realized by changing the thickness of each part.

(2) By reducing the thickness of the upper or middle heat pellets and increasing the thickness of the bottom insulation layer, the discharge time of the thermal battery can be improved.

(3) Reducing the thickness of the upper and middle heat pellets slightly prolong the activation time of the thermal battery.

(4) After the multi-objective optimization, the discharge time of the thermal battery can be prolonged by $4.08 \%$, while the activation time is only increased by $1.23 \%$.

(5) The deviation between the multi-objective optimization result and the CFD simulation result is less than $1 \%$, which verified the correctness of the optimization method.

Author Contributions: Conceptualization, H.-L.L. and X.-D.S.; Methodology, Q.L.; Software, Y.-Q.S.; Validation, Q.L.; Formal Analysis, H.-L.L.; Investigation, Q.L.; Resources, X.-D.S.; Data Curation, Y.-Q.S.; Writing-Original Draft Preparation, Q.L. and Y.-Q.S.; Writing-Review \& Editing, H.-L.L. and X.-D.S.; Visualization, Q.L. and Y.-Q.S.; Supervision, X.-D.S.; Project Administration, X.-D.S.; Funding Acquisition, X.-D.S. All authors have read and agreed to the published version of the manuscript.

Funding: This research was funded by Tianjin Major Science and Technology Project, grant number 019040012 and the Major Project of Ministry of Industry and Information Technology, grant number C019040004. The APC was funded by Intelligent Manufacturing Integrated Standardization Project.

Acknowledgments: This work is supported by Tianjin Major Science and technology projects (No. 91001190011) and the Shaanxi Natural Science Foundation (No. 2019JM-071).

Conflicts of Interest: The authors declared that they have no conflicts of interest to this work. We declare that we do not have any commercial or associative interest that represents a conflict of interest in connection with the work submitted.

\section{Nomenclature}

$\begin{array}{ll}C_{p} & \text { Heat Capacity, } \mathrm{J} \mathrm{kg}^{-1} \mathrm{~K}^{-1} \\ d & \text { thickness, } \mathrm{mm} \\ \Delta d & \text { the decrease of the thickness, } \mathrm{mm} \\ h & \text { convective heat transfer coefficient, } \mathrm{W} \mathrm{m}^{-2} \mathrm{~K}^{-1} \\ i_{A} & \text { current density, } \mathrm{A} \mathrm{m}^{-2} \\ i_{V} & \text { current per unit volume, } \mathrm{A} \mathrm{m}^{-3} \\ p & \text { the pressure of air, Pa } \\ P & \text { the size of population } \\ q & \text { energy density, } \mathrm{W} \mathrm{m} \mathrm{m}^{-3} \\ Q & \text { the size of genelation } \\ q_{c r} & \text { chemical reaction heat, } \mathrm{W} \mathrm{m} \mathrm{m}^{-3} \\ q_{j h} & \text { Joule heating, } \mathrm{W} \mathrm{m} \mathrm{m}^{-3} \\ \mathrm{R} & \text { resistivity, } \Omega \mathrm{m} \\ t & \text { time, } \mathrm{s}\end{array}$




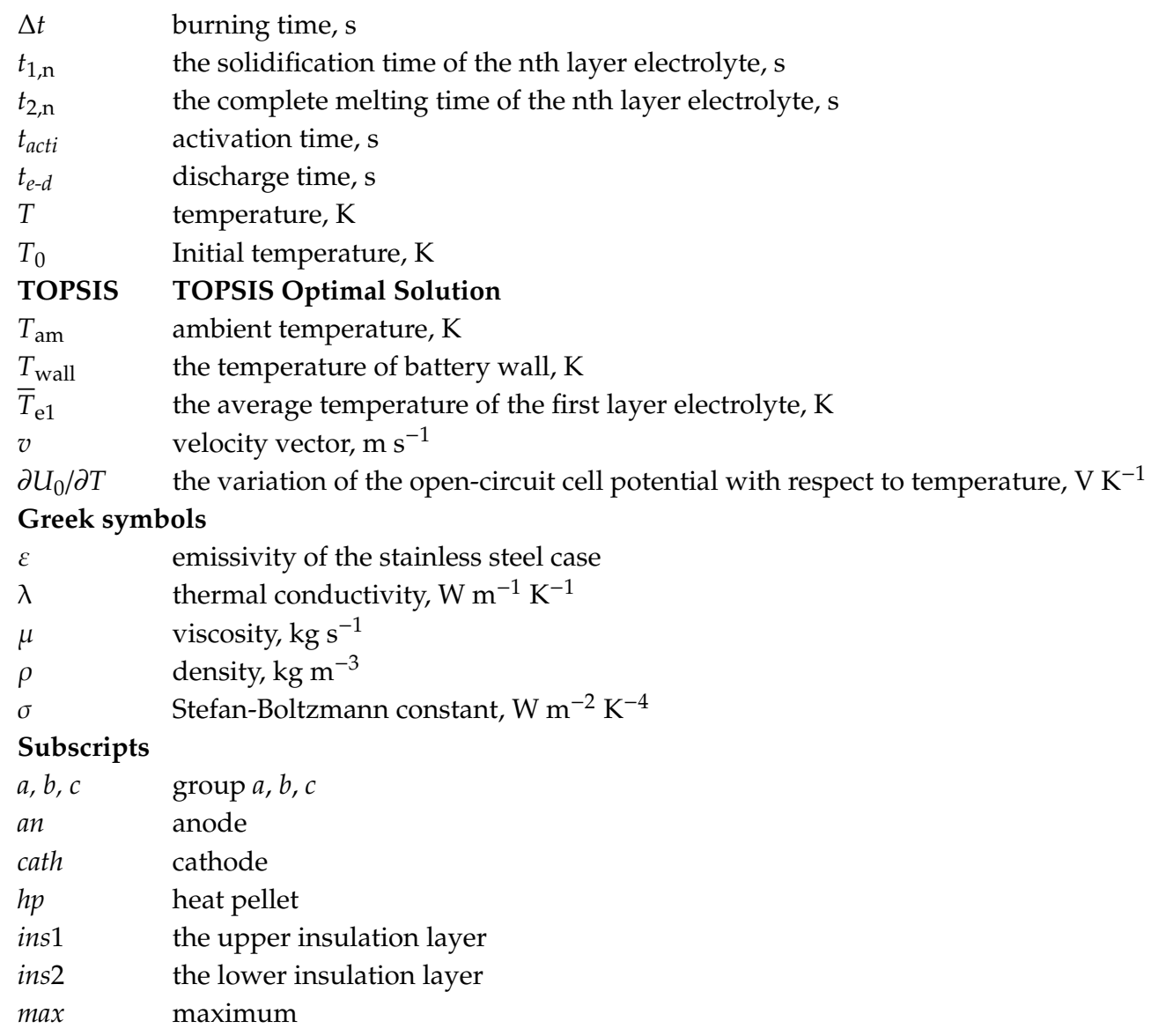

\section{References}

1. Guidotti, R.A.; Masset, P. Thermally activated ("thermal”) battery technology Part I: An overview. J. Power Sources 2006, 161, 1443-1449. [CrossRef]

2. Choi, P.; Mills, I.; Singhal, S.; Sanghadasa, M. A high power thermal battery based on high voltage cathode and stable electrolyte. In Proceedings of the 47th Power Sources Conference, Orlando, FL, USA, 13-16 June 2016; pp. 449-452.

3. Johnson, Z.; Pickett, D.; Wilkes, J.; Burns, B.; Preston, S. Inherently safe and stable high-temperature battery. In Proceedings of the 42th Power Sources Conference, Philadelphia, PA, USA, 12-15 June 2006; pp. $103-106$.

4. Guidotti, R.A.; Reinhardt, F.W.; Odinek, J. Overview of high-temperature batteries for geothermal and oil/gas borehole power sources. J. Power Sources 2004, 136, 257-262. [CrossRef]

5. Kang, B.; Xing, Y.H.; Zhao, Y.X.; Cao, J.J.; Lin, H.B. Study on effect of electrolyte systems on activation time of $\mathrm{FeS}_{2} / \mathrm{LiSi}$ thermal battery, Chinese. J. Power Sources 2015, 39, 2153-2154.

6. Kang, B.; Zhang, W.; Lin, H.; Xing, Y.; Zhao, J.; Wang, Y. Thermal transfer during the activation process in $\mathrm{LiSi} / \mathrm{FeS}_{2}$ thermal batteries. Chem. Res. Chin. Univ. 2016, 4, 665-668. [CrossRef]

7. Witt, B.; Zwink, B. Pushing 3D scanning laser doppler vibrometry to capture time varying dynamic characteristics. In Rotating Machinery, Vibro-Acoustics $\mathcal{E}$ Laser Vibrometry, Proceedings of the 36th IMAC, Orlando, FL, USA, 12-15 February 2018; Springer: Cham, Switzerland, 2018; Volume 7, pp. 111-121.

8. Koyuncu, T.; Alkan, A.; Sevim, K.; Yesilirmak, Y.; Unsal, E.; Gocmez, A. Modeling of pyrotechnic combustion behavior and temperature distribution for the ignition phase of thermal batteries. In Proceedings of the 48th Power Sources Conference, Denver, CO, USA, 11-14 June 2018; pp. 428-431.

9. Krieger, F.C.; Lennen, R. Practical miniaturization of the LCCM thermal battery using gas getters. In Proceedings of the 42th Power Sources Conference, Philadelphia, PA, USA, 12-15 June 2006; pp. 253-256.

10. Krieger, F.C.; Ding, M.S. Thermal battery Operating Gas Atmosphere Control and Heat Transfer Optimization; Army Research Laboratory: Adelphi, MD, USA, 2012. 
11. Yang, T.T.; Cai, L.; White, R.E. Mathematical modeling of the $\mathrm{LiAl} / \mathrm{FeS}_{2}$ high temperature battery system. J. Power Sources 2012, 201, 322-331. [CrossRef]

12. Haimovich, N.; Dekel, D.R.; Brandon, S. A simulator for system-level analysis of heat transfer and phase-change in thermal batteries: II. Multiple-cell simulations. J. Electrochem. Soc. 2015, 162, A350-A362. [CrossRef]

13. Chen, C.F.; Li, H.Y.; Hong, C.W. Molecular dynamics analysis of high-temperature molten-salt electrolytes in thermal batteries. Comput. Mater. Contin. 2015, 46, 145-163.

14. Jeong, M.G.; Cho, J.-H.; Lee, B.J. Heat transfer analysis of a high-power and large-capacity thermal battery and investigation of effective thermal model. J. Power Sources 2019, 424, 35-41. [CrossRef]

15. Ruan, H.J.; Jiang, J.C.; Sun, B.X.; Sun, X.J.; He, X.T.; Zhao, K.J. An optimal internal-heating strategy for lithium-ion batteries at low temperature considering both heating time and lifetime reduction. Appl. Energy 2019, 256, 113797. [CrossRef]

16. Zhang, L.Q.; Wang, L.X.; Hinds, G.; Lyu, C.; Zheng, J.; Li, J.F. Multi-objective optimization of lithium-ion battery model using genetic algorithm approach. J. Power Sources 2014, 270, 367-378. [CrossRef]

17. Shui, L.; Chen, F.Y.; Garg, A.; Peng, X.B.; Bao, N.S.; Zhang, J. Design optimization of battery pack enclosure for electric vehicle. Struct. Multidiscip. Optim. 2018, 4, 331-347. [CrossRef]

18. Liu, H.L.; Shi, H.B.; Shen, H.; Xie, G.N. The performance management of a Li-ion battery by using tree-like mini-channel heat sinks: Experimental and numerical optimization. Energy 2019, 189, 116150. [CrossRef]

19. Roger, C.R.; Yen, S.H.; Ramanathan, K.G. Temperature variation of total hemispherical emissivity of stainless steel AISI 304. J. Opt. Soc. Am. 1979, 69, 1384-1390. [CrossRef]

20. Park, B.J.; Kim, J.Y.; Ha, S.H.; Cho, J.H. Performance estimation based on 4D lookup table interpolating and unit cell discharge tests for thermal battery. J. Korean Inst. Electr. Electron. Mater. Eng. 2017, 30, 393-400.

21. Haimovich, N.; Dekel, D.R.; Brandon, S. A simulator for system-level analysis of heat transfer and phase change in thermal batteries: I. Computational approach and single-cell calculations. J. Electrochem. Soc. 2009, 156, A442-A453. [CrossRef]

22. Yazdani-Asrami, M.; Taghipour-Gorjikolaie, M.; Song, W.J.; Zhang, M.; Yuan, W.J. Prediction of nonsinusoidal AC loss of superconducting tapes using artificial intelligence-based models. IEEE Access 2020, 8, 207287-207297. [CrossRef]

23. Hwang, C.L.; Yoon, K. Methods for Multiple Attribute Decision Making; Springer: Berlin/Heidelberg, Germany, 1981.

24. Yoon, K. A reconciliation among discrete compromise solutions. J. Oper. Res. Soc. 1987, 38, 277-286. [CrossRef]

25. Hwang, C.L.; Lai, Y.J.; Liu, T.Y. A new approach for multiple objective decision making. Comput. Oper. Res. 1993, 20, 889-899. [CrossRef]

26. Ge, Y.; Wang, S.C.; Liu, Z.C.; Liu, W. Optimal shape design of a mini-channel heat sink applying multi-objective optimization algorithm and three-dimensional numerical method. Appl. Therm. Eng. 2019, 148, 120-128. [CrossRef]

Publisher's Note: MDPI stays neutral with regard to jurisdictional claims in published maps and institutional affiliations.

(C) 2020 by the authors. Licensee MDPI, Basel, Switzerland. This article is an open access article distributed under the terms and conditions of the Creative Commons Attribution (CC BY) license (http://creativecommons.org/licenses/by/4.0/). 